\title{
Lobação do fígado e distribuição intraparenquimal da veia porta no preá (Galea spixii Wagler, 1831)
}

\author{
Gleidson Benevides de Oliveira ${ }^{1 *}$ \\ Márcio Nogueira Rodrigues ${ }^{2}$ \\ Roberto Sávio Bessa Silva ${ }^{1}$ \\ José Fernando Gomes de Albuquerque ${ }^{3}$ \\ André Menezes do Vale ${ }^{1}$ \\ Carlos Eduardo Bezerra de Moura ${ }^{4}$ \\ Moacir Franco de Oliveria ${ }^{1}$ \\ ${ }^{1}$ PPG em Ciência Animal, Universidade Federal Rural do Semiárido \\ km 47, BR 110, CEP 59625-900, Mossoró - RN, Brasil \\ ${ }^{2}$ Faculdade de Medicina Veterinária e Zootecnia, Universidade de São Paulo, São Paulo - SP, Brasil \\ ${ }^{3}$ Departamento de Ciências Animais, Universidade Federal Rural do Semiárido, Mossoró - RN, Brasil \\ ${ }^{4}$ Centro de Biociências, Universidade Federal do Rio Grande do Norte, Natal - RN, Brasil \\ *Autor para correspondência \\ gleidson_benevides@hotmail.com
}

Submetido em 29/03/2011

Aceito para publicação em 28/06/2011

\section{Resumo}

A lobação do fígado e a distribuição intraparenquimal da veia porta foi descrita com base em estudos realizados em 10 exemplares de preás. A veia porta foi canulada e perfundida com solução de acetato de vinil e o fígado imerso em solução de ácido sulfúrico para obtenção de moldes vasculares. O fígado apresentou cinco lobos, que foram denominados lobos lateral esquerdo, medial esquerdo, quadrado, lateral direito, medial direito e caudado com seus processos papilar e caudado. A veia porta, ao penetrar na fissura portal, dividia-se em dois ramos, um direito e outro esquerdo. O ramo direito emitia ramos isolados ou por tronco comum aos lobos lateral direito e ao processo caudado do lobo caudado. Já o ramo principal esquerdo da veia porta dividia-se em dois outros ramos. Um dos ramos formava um tronco comum para os lobos lateral esquerdo, medial esquerdo e quadrado, e os dois últimos em via comum. O segundo ramo destinava-se ao ramo medial direito. Logo após sua formação, o tronco comum do ramo principal esquerdo emite pequenos ramos vasculares para o processo papilar do lobo caudado.

Palavras-chave: Fígado, Galea spixii, Roedor, Veia porta

\section{Abstract}

Liver lobation and intraparenchymal distribution of the portal vein in Brazilian guinea pig (Galea spixii Wagler, 1831). Liver lobation and intraparenchymal distribution of the portal vein was described according to studies performed in 10 specimens of Brazilian guinea pigs. The portal vein was cannulated and perfused 
with a vinyl acetate solution and the liver immersed in sulfuric acid solution to obtain vascular casts. The liver presented five lobes, which were named left lateral, left medial, square, right lateral, right medial, and caudate lobes with caudate and papillary processes. The portal vein, entering the portal fissure, divided into two branches, the right and left ones, as it entered the portal fissure. The right branch emitted isolated or common trunk branches to the right lateral lobe and to the caudate process of caudate lobe. On its turn, the left main branch of portal vein divided into two other branches. One of them formed a common trunk for the left lateral, left medial, and square lobes, being the two last ones in a common way. The second branch coursed towards to the right medial branch. Just after its formation, the common trunk of the left main branch emits small vascular branches to the papillary process of caudate lobe.

Key words: Galea spixii, Liver, Portal vein, Rodent

\section{Introdução}

Os roedores são classificados como animais que pertencem à ordem Rodentia, palavra derivada do latim rodere (roer). A principal característica que os unem é a presença de dentes incisivos bem proeminentes que crescem continuamente. A classificação quanto à espécie dá-se através de caracteres morfológicos como pelagem, dentição, tamanho das orelhas e dos olhos, presença e tamanho da cauda (BRASIL, 2002). Segundo o autor, o preá é um roedor sinantrópico silvestre pertencente à subordem Hystricognathi, família Caviidae que habita o semi-árido nordestino. Moojen (1952) descreve que este roedor apresenta superfície dorsal acinzentada, com um tom brunáceo e superfície ventral branca, possuindo ainda uma mancha infra-ocular branca, com hábito crepuscular, percorrendo trilhas ao anoitecer ou ao amanhecer e que vive em bandos de seis a quinze indivíduos, especialmente nas pequenas moitas das caatingas ralas e entre as cactáceas e bromeliáceas fazendo os ninhos em buracos ou sob galhos secos.

$\mathrm{O}$ estudo da morfologia em animais selvagens é sempre uma fonte crescente de informações, especialmente quando se trata de espécies que apresentam algum potencial zootécnico ou valor biológico, como é o caso do preá, roedor da fauna nordestina com grande importância econômica e social, uma vez que sua carne é utilizada como fonte de proteína animal, em comunidades carentes ou por hábitos culturais do nordestino. Com base no exposto e considerando a escassez de dados referentes à morfologia do preá, em especial sobre a vascularização porta-hepática e a morfologia do fígado, desenvolveuse este estudo, que descreve a lobação do fígado e a distribuição intraparenquimal da veia porta no preá.

\section{Material e Métodos}

Para desenvolvimento deste estudo foram utilizados 10 preás, todos machos adultos, que vieram a óbito por causas naturais e que se encontravam congelados. Os animais foram obtidos no Centro de Multiplicação de Animais Silvestres - CEMAS/UFERSA, situado no município de Mossoró, no Rio Grande do Norte, licenciado junto ao IBAMA como criadouro cientifico registrado sob o número 14.78912. Os animais foram descongelados e incisados medianamente para abertura da cavidade abdominal, sendo a veia porta canulada no sentido cranial para injeção de solução de acetato vinil de cor azul. Os moldes vasculares do fígado foram obtidos por processo de corrosão em solução de ácido sulfúrico a $30 \%$, sendo as estruturas denominadas com base na nomenclatura adotada pelo International Committee on Veterinary Gross Anatomical Nomenclature (2005).

\section{Resultados}

\section{Morfologia do fígado do preá}

O fígado dos mamíferos, normalmente é descrito como um órgão que possui topografia e lobação variável. $\mathrm{Na}$ espécie, este órgão é lobado, apresentando os lobos hepáticos: lateral esquerdo, medial esquerdo, quadrado, medial direito, lateral direito e caudado, com seus processos caudado e papilar, verificando-se ainda a presença da vesícula biliar (Figura 1A e 1B). 


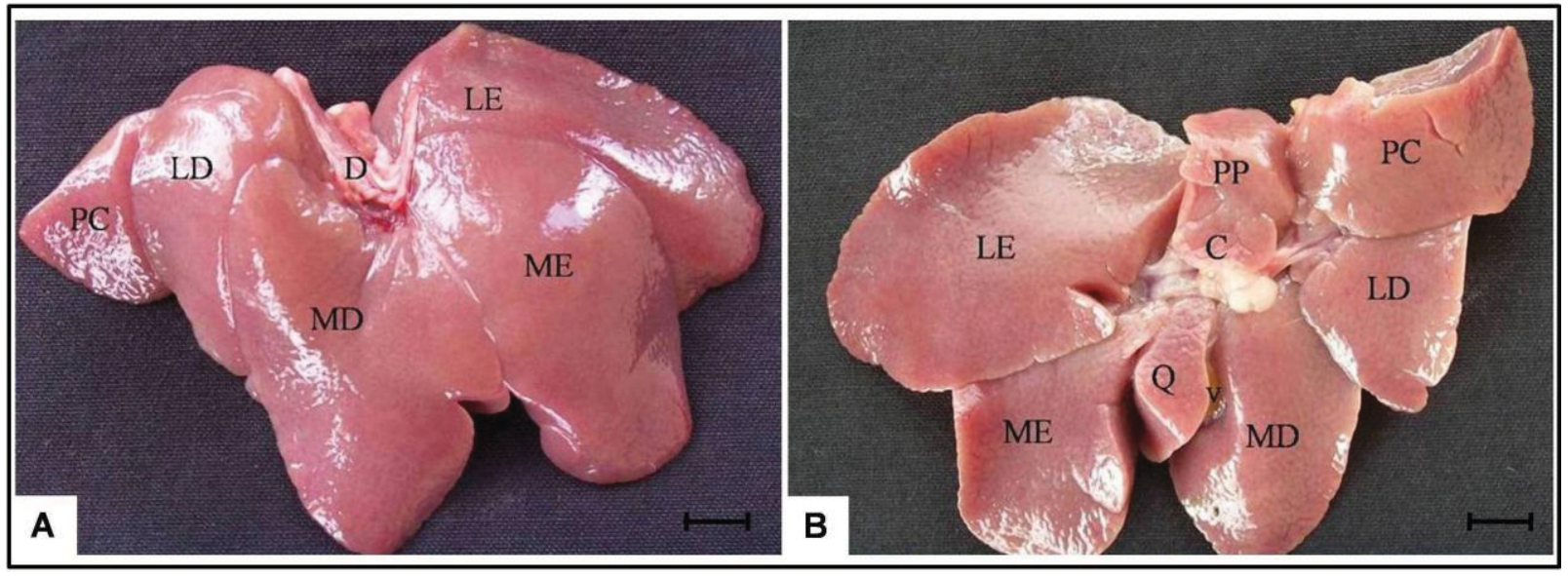

FIGURA 1: Fotografia do fígado de preá. Em (A) tem-se uma vista da face diafragmática. Identificam-se os lobos laterais direito (LD) e esquerdo (LE), mediais direito (MD) e esquerdo (ME), quadrado (Q), processo caudado do lobo caudado (PC) e ainda o diafragma (D).Em (B) tem-se uma vista da face visceral. Estão identificados os lóbulos: laterais direito (LD) e esquerdo (LE), mediais direito (MD) e esquerdo (ME), quadrado (Q), caudado (C), os processo papilar (PP) e caudado (PC) e a vesícula biliar (v). Barra de escala: 10cm. CEMAS 2009.

\section{Distribuição intraparenquimal da veia}

\section{porta}

As análises dos moldes vasculares obtidos a partir da injeção de acetado de vinil no sistema porta-hepático, permitiram que fosse verificado o comportamento da veia porta no preá ao penetrar no fígado. Em $100 \%$ dos animais estudados observou-se que a mesma dividia-se em um ramo direito e outro esquerdo, sendo o esquerdo mais calibroso (Figura 2A).

O ramo direito emitia ramos isolados para o lobo lateral direito e para o processo caudado do lobo caudado em oito animais (80\%) (Figura 2B). Destes, $75 \%$ emitiam um ramo para o processo caudado e em um único animal o ramo direito emitia dois $(12,5 \%) \mathrm{e} / \mathrm{ou}$ três $(12,5 \%)$ ramos para este lobo. Nos demais animais

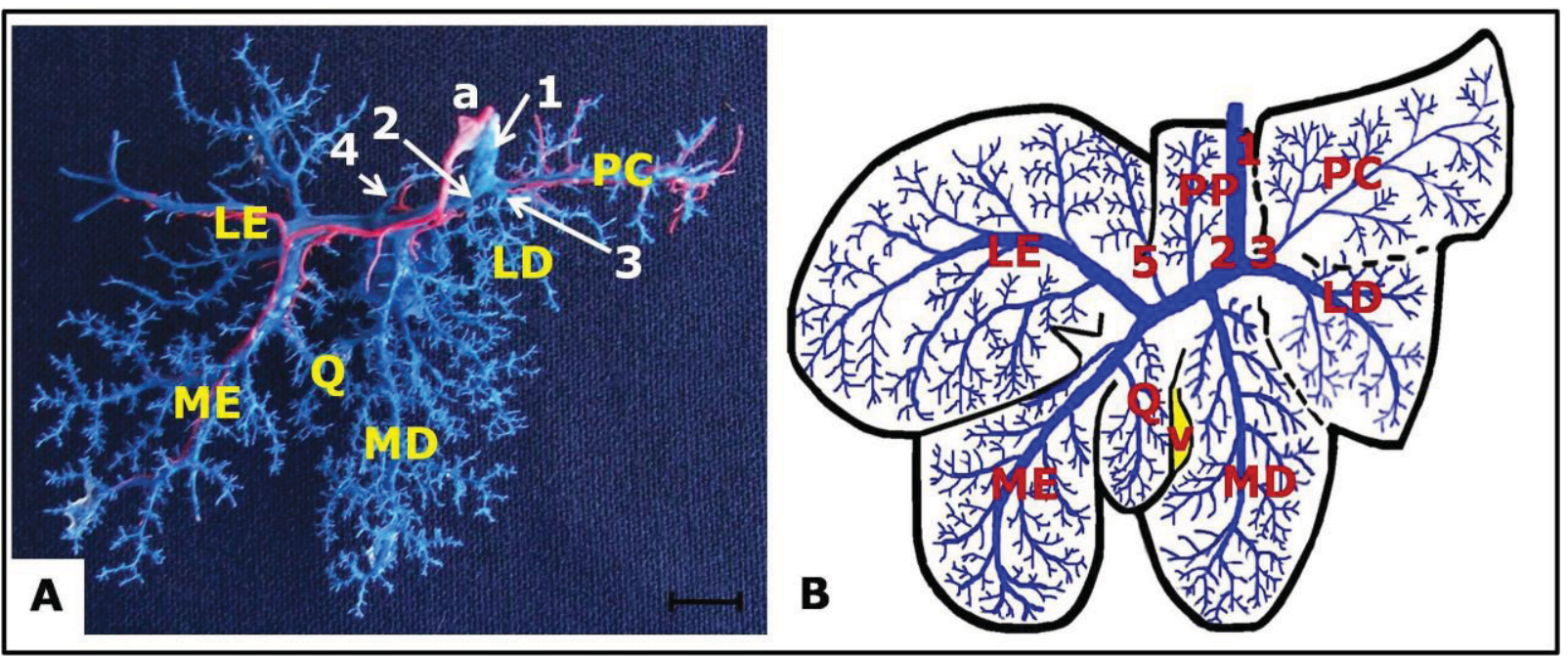

FIGURA 2: Distribuição intraparenquimal da veia porta (1) no preá. Em A, observa-se os ramo esquerdo (2) distribuindo-se pelos lobos lateral esquerdo (LE), medial esquerdo (ME), quadrado (Q), medial direito (MD) e um ramo (4) para o processo papilar (PP) do lobo caudado; e o ramo direito (3) distribuindo-se em via comum aos lobos lateral direito (LD) e processo caudado (PC). Observa-se ainda a artéria hépática (a). Em B, a representação esquemática do molde vascular, identificando-se os ramos esquerdo (2) e direito (3) da veia porta e ainda o ramo destinado a porção dorsal do lobo lateral esquerdo (5) e a vesícula biliar (v). Barra de escala: 10cm. CEMAS, 2009. 
(20\%), os lobos lateral direito e o processo caudado receberam o sangue portal por tronco comum (Figura 3A e 3C). Ressalta-se que quando da presença desse tronco comum, o lobo lateral direito ainda recebia um ramo que saia diretamente do ramo principal direito.

O ramo principal esquerdo da veia porta dividia-se, em todos os espécimes estudados, em dois outros ramos. Um dos ramos formava um tronco comum para os lobos lateral esquerdo, medial esquerdo e quadrado, os dois últimos em via comum. $\mathrm{O}$ segundo ramo destinava-se ao ramo medial direito (Figura 3B e 3C). Logo após sua formação, o tronco comum do ramo principal esquerdo emitia de um a dois pequenos vasos para o processo papilar, em $80 \%$ e $20 \%$ dos casos, respectivamente.

Já o lobo quadrado, recebeu o sangue portal em via comum com o lobo medial esquerdo. No entanto, em $50 \%$ dos animais amostrados esse tronco comum emitia um único ou dois ramos.

Para o lobo lateral esquerdo, além do vaso oriundo da bifurcação do tronco comum, especificado, este lobo recebia um outro ramo, o ramo dorsal, que se distribuía para atender a porção dorsal do fígado, fato observado em $80 \%$ dos moldes vasculares.

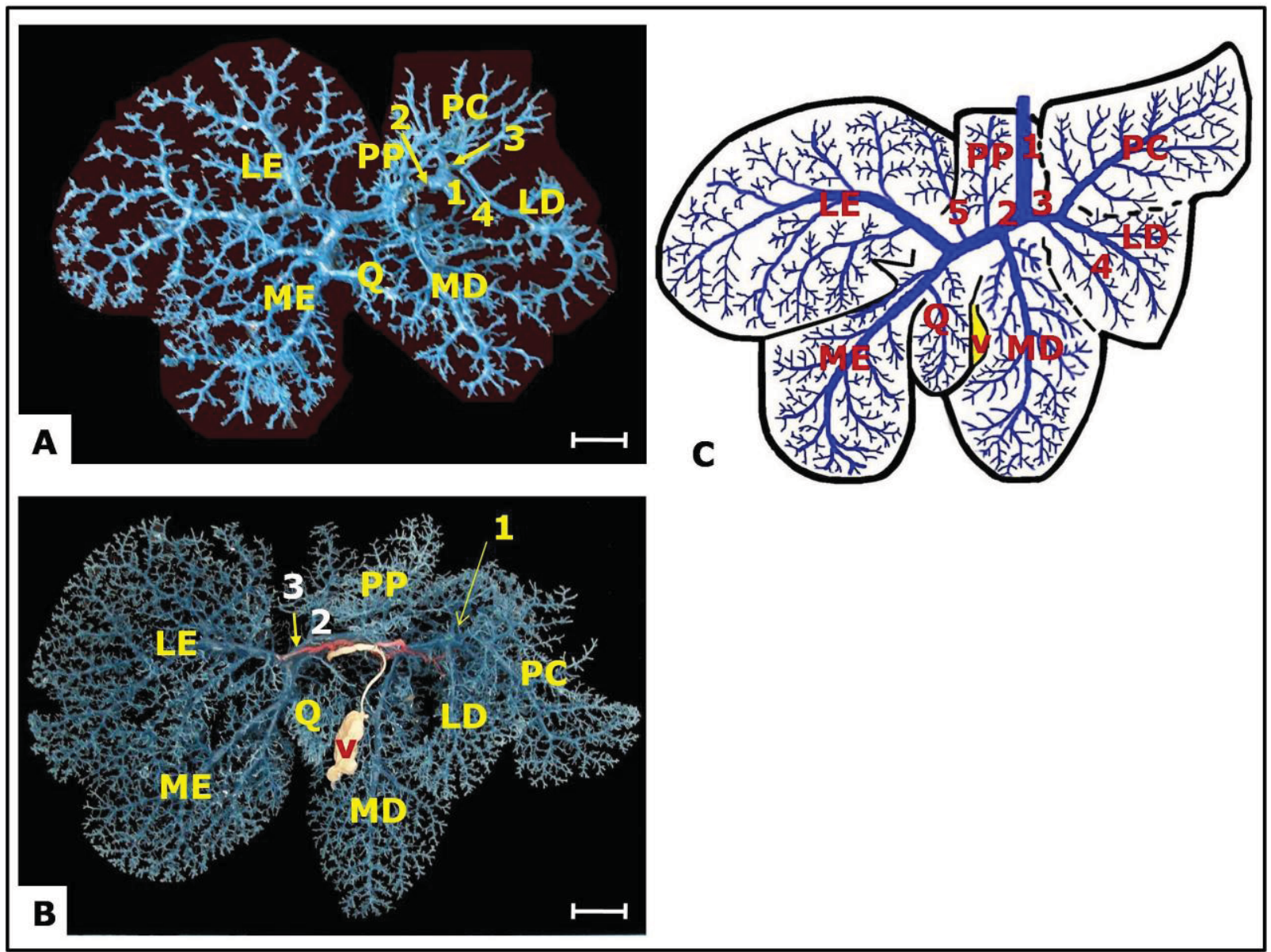

FIGURA 3: Distribuição intraparenquimal da veia porta no fígado do preá, em vista da face visceral do órgão. Em A, identificase o ramo direito (3) da veia porta (1) distribuindo-se em tronco comum aos lobos hepáticos lateral direito (LD) e processo caudado do lobo caudado (PC) e um ramo que sai do ramo principal direito destinando-se ao lobo hepático lateral direito. Em B, identifica-se a vesícula biliar (v) e o tronco comum (3), emitido pelo ramo esquerdo, destinando-se aos lobos lateral e medial esquerdo (LE e ME) e quadrado (Q), bem como ao processo papilar do lobo caudado (PP). Em C, a representação esquemática da distribuição intraparenquimal da veia porta do preá. Identifica-se a veia porta (1) e seus ramos direito (3) e esquerdo (2). Tem-se ainda um ramo que parti do ramo direito (4) e um ramo dorsal que sai do ramo principal esquerdo (5) destinado aos lobos lateral direito e lateral esquerdo, respectivamente. Barra de escala: 10cm. CEMAS 2009. 


\section{Discussão}

\section{Morfologia do fígado do preá}

O fígado do preá a exemplo do que descreve Krahmer e Schröder (1979), apresenta uma face diafragmática convexa e uma face visceral côncava, bastante distintas. Esta última contendo o hilo hepático composto por vasos sanguíneos, nervos e ductos biliares. Embora, o autor refira-se a presença de gânglios linfáticos na composição do hilo hepático, macroscopicamente, em preás não se observou sua ocorrência.

Quanto à sua lobação, o fígado esteve dividido em cinco lobos distintos, denominados conforme Nômina Anatômica Veterinária (2005). Pesquisadores como Barone et al. (1973), Cooper e Schiller (1975), Souza et al. (1994), Menezes et al. (2001) e Azevêdo et al. (2008) descrevem a lobação do fígado de coelhos, cobaias, quatis e cutias, respectivamente, e afirmam que este é lobado e apresenta os lobos lateral esquerdo, medial esquerdo, quadrado, medial direito, lateral direito e caudado, com seus processos caudado e papilar, resultados que se assemelham aos encontrados para o preá. Entretanto, Souza et al. (2007), ao descreverem a artéria hepática da capivara, afirmam que o fígado tem a mesma lobação, observada para os animais referenciados, mas ressaltam que os lobos quadrado e medial direito são quase totalmente fusionados. Torna-se importante destacar que nos estudos com quatis, cutias e capivaras os autores relatam a presença da vesícula biliar, assim como observado no preá.

\section{Distribuição intraparenquimal da veia porta}

A veia porta do preá ao penetrar na fissura portal divide-se em dois ramos, direito e esquerdo, comportamento, semelhante ao descrito por Miglino et al. (1997), na capivara, Silva et al. (2000), em ratos, Menezes et al. (2001), na cutia, e Menezes et al. (2003), na raposa, em estudos com a distribuição intraparenquimal da veia porta. Todavia, D'Errico et al. (1982), no ratão-do-banhado, e Menezes et al. (2001), na cutia, citam a divisão da veia porta em ramos direito, intermédio e esquerdo, descrição não observada no preá.
Ao discorrer sobre a divisão e distribuição intraparenquimal da veia porta, Cooper e Schiller (1975) citam em cobaias, que no fígado, esta se divide em quatro troncos principais: um para o lobo direito; outro, à direita, para o lobo quadrado; um tronco pequeno para o lobo caudado; e um grande tronco comum, à esquerda, para os lobos quadrado e esquerdo.

No Galea spixii a distribuição intraparenquimal da veia porta no fígado, diferencia-se aos resultados relatados por Menezes et al. (2001) na cutia, quando os mesmos verificaram que o ramo esquerdo bifurca-se após ceder finos ramos para o processo papilar do lobo caudado, dando um ramo para o lobo medial direito e outro, em tronco comum, aos lobos medial esquerdo, quadrado e lateral esquerdo. Os autores citam que o ramo direito subdivide-se mais frequentemente em dois ramos, destinados aos lobos lateral direito e processo caudado do lobo caudado, semelhante ao que acontece no preá. Quando da presença do ramo médio este direciona-se para o lobo medial direito. No preá, o ramo médio não esteve presente. $\mathrm{O}$ autor cita ainda um ramo para o lobo medial direito oriundo do ramo principal direito, fato não encontrado no preá.

Miglino et al. (1997), ao estudarem a distribuição intraparenquimal da veia porta na capivara, comentam que o ramo direito divide-se, inicialmente, em dois componentes, sendo um destinado ao lobo medial direito e outro relativo ao processo caudado do lobo caudado. No preá, observou-se que o ramo principal direito da veia porta não enviou ramos para o lobo medial direito, responsabilizando-se por este lobo o ramo venoso oriundo do ramo esquerdo da veia porta. Os autores ainda citam que o ramo esquerdo da veia porta divide-se em dois, sendo um tronco comum aos vasos destinados aos lobos quadrado e medial esquerdo, e outro componente isolado relativo ao lobo lateral esquerdo, diferindo dos resultados obtidos para preá.

Silva et al. (2000) relataram, em ratos, que o ramo direito da veia porta, se subdivide em três ramos, irrigando exclusivamente o lobo direito, enquanto o ramo esquerdo, subdivide-se em cinco ramos responsáveis pela irrigação do restante do parênquima hepático. Menezes et al. (2004), estudando a veia porta da raposa, concluem que o ramo direito cede um pequeno vaso 
dirigido ao processo caudado do lobo caudado e um tronco comum aos vasos destinados ao lobo lateral direito, podendo o processo caudado receber um ramo do tronco da veia porta antes de sua divisão nos ramos principais, diferindo dos resultados encontrados no preá, pois em nenhum momento o processo caudado do lobo caudado recebeu ramos diretamente da veia porta antes de ocorrer sua divisão. Citam ainda os autores, que o ramo esquerdo da veia porta divide-se num tronco comum aos lobos lateral esquerdo, medial esquerdo e quadrado, sendo que os dois últimos lobos recebem vasos simultaneamente, e um único vaso destinado ao lobo medial direito. Tal situação também foi observada na distribuição intraparenquimal da veia porta no preá. Os mesmos ressaltam que o ramo esquerdo da veia porta emite um ramo para o processo papilar e pode emitir um ramo para o lobo lateral direito, descrição não verificada na espécie em discussão.

A partir dos resultados, é possível concluir que o fígado do preá apresenta padrão de lobação similar a de roedores, como o coelho, a cobaia e cutia, apresentando os lobos lateral esquerdo, medial esquerdo, quadrado, medial direito, lateral direito e lobo caudado, com seus processos papilar e caudado.

A veia porta ao inserir-se ao fígado do preá bifurca-se em um ramo direito e outro esquerdo; os lobos lateral direito e o processo caudado do lobo caudado recebem sangue do ramo direito da veia porta, enquanto os lobos medial direito e o processo papilar do lobo caudado recebem sangue do ramo esquerdo, enquanto os lobos lateral esquerdo, medial esquerdo e quadrado recebem sangue do ramo esquerdo através de um tronco comum.

\section{Referências}

AZEVÊDO, L. M.; CARVALHO, M. A. M.; MENEZES, D. J. A.; MACHADO, G. V.; SOUZA, A. A. R.; XAVIER, F. G. Distribuição intraparenquimal da artéria hepática em cutias (Dasyprocta sp., Rodentia). Brazilian Journal of Veterinary Research and Animal Science, São Paulo, v. 45, n. 1, p. 5-10, 2008.

BARONE, R.; PAVAUX, C.; BUN, P. C.; CUQ, P. Atlas d'anatomie du lapin. Paris: Masson, 1973. 219 p.

BRASIL - Fundação Nacional de Saúde (FUNASA). Manual de controle de roedores. Brasília: Ministério da Saúde, Fundação Nacional de Saúde, 2002. 132 p.
COOPER, G.; SCHILlER, A. L. Anatomy of the guinea pig. Cambridge: Harvard University Press, 1975. 417 p.

D'ERRICO, M. A.; PRADA, L. L. S.; FERNANDES FILHO, A. Contribuição ao estudo do comportamento da veia porta no fígado do ratão-do-banhado (Myocastor cozypus). In: CONGRESSO PERNAMBUCANO DE MEDICINA VETERINÁRIA, I, 1982, Anais... Recife: CPMV, 1982. p. 127.

INTERNATIONAL COMMITTEE ON VETERINARY GROSS ANATOMICAL NOMENCLATURE. Nomina anatomica veterinária. 5. ed. Knoxville: World Association on Veterinary Anatomist, 2005. 190 p.

KRAHMER, R.; SCHRÖDER, L. Anatomía de los animales domésticos. Zaragoza: Editorial Acribia, 1979. 144 p.

MENEZES, D. J. A.; CARVALHO, M. A. M.; MACHADO, G. V.; CAVALCANTE FILHO, M. F. Distribuição intraparenquimal da veia porta hepática na cutia (Dasyprocta aguti - Rodentia: Mammalia). Archives of Veterinary Science, Curitiba, v. 6, n. 2, p.1-7, 2001.

MENEZES, D. J. A.; YASSAKI, J. K. M.; MEDEIROS, G. X.; MASUKO, T. S.; GOMES, A. A. B; CARVALHO, M. A. M. Distribuição intraparenquimal da veia porta na raposa (Dusicyon vetulus). International Journal of Morphology, Temuco, v. 22, n. 1, 2003.

MIGLINO, M. A.; SOUZA, W. M.; DIDIO, L. J. A. Distribuição da veia porta no fígado da capivara. Brazilian Journal of Veterinary Research and Animal Science, São Paulo, v. 34, n. 1, p. 9-11, 1997.

MOOJEN, J. Os roedores do Brasil. Rio de Janeiro: Instituto Nacional do Livro, Ministério de Educação e Saúde, 1952. 214 p.

SILVA, V. A. S.; EPAMINONDAS, W. A.; MENESCAL, R. E. S.; BRITO, M. V. H. Estudo angioarquitetônico da veia porta em ratos. Revista Paraense de Medicina, Belém, v. 14, n. 2, p. 29-32, 2000. SOUZA, W. M.; MIGLINO, M. A.; D’ERRICO, A. A.; DIDIO, L. J. A. As vias bilíferas do quati (Nasua narica). Unimar Ciências, Marília, v. 3, p. 23-29, 1994.

SOUZA, W. M.; SOUZA, N. T. M.; CARVALHO, R. G.; CORREA, C. N. Topografia da artéria hepática no fígado de capivara. Ciência Rural, Santa Maria, v. 37, n. 1, p. 141-145, 2007. 